\title{
Epigenetics Evolution and Replacement Histones: Evolutionary Changes at Drosophila H4r
}

\author{
Yoshinori Yamamoto', Toru Watanabe ${ }^{1}$, Mayu Nakamura1, Norikazu Kakubayashi'1,2, Yusuke Saito ${ }^{1}$ and Yoshinori Matsuo ${ }^{1-4 *}$ \\ ${ }^{1}$ Laboratory of Adaptive Evolution, Faculty of Integrated Arts and Sciences, Minamijosanjima-cho 1-1, Tokushima University, Tokushima 770-8502, Japan \\ ${ }^{2}$ Graduate School of Integrated Arts and Sciences, Minamijosanjima-cho 1-1, Tokushima University, Tokushima 770-8502, Japan \\ ${ }^{3}$ Institute of Socio-Arts and Sciences, Tokushima University, Minamijosanjima-cho 1-1, Tokushima 770-8502, Japan \\ ${ }^{4}$ Graduate School of Science and Technology, Tokushima University, Minamijosanjima-cho 2-1, Tokushima 770-8506, Japan
}

\begin{abstract}
Histone 4 replacement $(\mathrm{H} 4 \mathrm{r})$ can replace replication-dependent $\mathrm{H} 4$ in Drosophila. To study the evolution of epigenetic mechanisms, the $\mathrm{H} 4$ and $\mathrm{H} 4 \mathrm{r}$ genes from 14 Drosophila species were compared with regard to gene arrangement, codon bias and flanking sequences. Although the amino acid sequences of $\mathrm{H} 4$ and $\mathrm{H} 4 \mathrm{r}$ are identical or nearly identical, the gene structures are quite different. The $\mathrm{H} 4 \mathrm{r}$ gene is a single copy gene located 3R88C9 in $D$. melanogaster between punt and CEP78K, as it is in 11 closely related Drosophila species, but not in the three distantly related species. The $\mathrm{H} 4 \mathrm{r}$ gene, unlike the $\mathrm{H} 4$ gene, has two introns and generates polyadenylated transcripts. The codon usage bias at particular sites differed between $\mathrm{H} 4 \mathrm{r}$ and $\mathrm{H} 4$. The $\mathrm{H} 4 \mathrm{r}$ gene had more $\mathrm{GC}$ pairs at $3^{\text {rd }}$ codon position. Strongly conserved signal sequence was not found in the 5'-region or 3'-region of the $\mathrm{H} 4 \mathrm{r}$ gene. These results suggested that the post transcriptional process such as modifying histone at or after translation will be important for replacing histones and remodeling the chromatin. The evolutionary changes that affect gene structure and codon usage might be a key step to develop epigenetic systems by replacement histones.
\end{abstract}

Keywords: Replacement histone; H4r; Drosophila; Epigenetics

\section{Introduction}

Knowledge about histone variants and histone modifications has become very important for studying topics in cell biology such as gene expression, DNA replication, development, cell memory, and chromatin remodeling [1-3]. New information in these fields might be also helpful for understanding quantitative genetics and phenotypic evolution [4-10]. Gene structures of replication-dependent histones (RDHs) and replication-independent histones (RIHs) were studied in a broad range of the species [11-12]. However, detailed comparisons between these two types of histone genes with regard to genome organization, control region, and codon usage have not been published. The differences could be related to the function of a specific histone.

Histone 4 is a small (about 102 amino acids) and highly conserved protein [13]. $\mathrm{H} 4$ binds $\mathrm{H} 3$ to form the $\mathrm{H} 3-\mathrm{H} 4$ dimer, which is then assembled into the histone core of the nucleosome [13]. In Drosophila, there are some histone variants; for example, $\mathrm{H} 3.3$ is a $\mathrm{H} 3$ variant [14] but there is no such variant for $\mathrm{H} 4$. However, a replacement $\mathrm{H} 4$ (H4r) that replaces $\mathrm{H} 4$ has been reported [15]. These two types of histone 4, $\mathrm{H} 4$ and $\mathrm{H} 4 \mathrm{r}$, have identical amino acid sequences [15]. This is probably because the $\mathrm{H} 4$ is highly conservative and even a single amino acid substitution was not permitted during the evolution of the two types. This identity is why $\mathrm{H} 4 \mathrm{r}$ is called a replacement histone not a histone variant.

In Drosophila, a single unit that contains five $\mathrm{RDH}$ genes $(\mathrm{H} 1, \mathrm{H} 2 \mathrm{~A}$, $\mathrm{H} 2 \mathrm{~B}, \mathrm{H} 3$, and $\mathrm{H} 4$ ) is repeated in tandem in a large gene cluster [16-23]. Therefore, each of the RDH genes exists in multiple copies (about 110 copies) [24-25]. Notably, the H4r gene structure differs substantially from the $\mathrm{H} 4$ gene structure; the $\mathrm{H} 4 \mathrm{r}$ gene is a single copy gene with two introns that produces transcripts with a poly(A) tail $[11,15,26]$. H4 and $\mathrm{H} 4 \mathrm{r}$ are expressed at different points in the cell cycle; however, it is not yet completely clear how or whether the obvious differences in gene structure relate to the specific function of each gene type.

Here, the H4r genes from 14 Drosophila species were examined to explore the functional significance of the $\mathrm{H} 4 \mathrm{r}$ gene. The $\mathrm{H} 4 \mathrm{r}$ genes from three Drosophila species (D. mauritiana, D. erecta, and $D$. orena) were newly cloned and sequenced. The genome arrangement, gene structure, and nucleotide sequences in the 5'-region, the coding region, and the 3'-region of the H4r genes were compared among 14 Drosophila species to clarify the roles of these histone replacements. Analyzing the mode and rate of evolution of these replacement histones should illuminate their contributions to phenotypic changes in these organisms during evolution.

\section{Materials and Methods}

\section{PCR and cloning}

Drosophila strains from D. mauritiana, D. erecta, and D. orena were donated by Kyushu University. A DNA extraction kit (Sepa Gene Kit, Sanko Junyaku, Co., Ltd.) was used to extract genomic DNA from Drosophila larvae. PCR reactions were conducted with Takara Ex Taq as follows [27]: for D. mauritiana and D. orena, denaturation occurred at $94^{\circ} \mathrm{C}$ for $1 \mathrm{~min}$, annealing at $54^{\circ} \mathrm{C}$ for $2 \mathrm{~min}$, followed by polymerization at $70^{\circ} \mathrm{C}$ for $2 \mathrm{~min}$ with extension for $5 \mathrm{sec}$ for 40 cycles. For D. erecta, the conditions for PCR were the same as above except that the annealing temperature was $53^{\circ} \mathrm{C}$. The primers used for PCR were 5'-TTTGTCGCAACGGG-3' (H4rF) and 5'-TGTGCTCCCTAAGC-3' (H4rR). The locations of primers for cloning of the H4r genes are shown in Figure 1. Plasmid vector PCR2.1 (Invitrogen) was used to clone each PCR product.

\section{Determination of nucleotide sequence}

Nucleotide sequence was determined with a Dye Terminator sequence kit (Applied Biosystems) and an ABI 310 sequencer [28]. The sequencing strategy for the histone gene region is depicted

*Corresponding author: Yoshinori Matsuo, Graduate School of Science and Technology, Tokushima University, Minamijosanjima-cho 2-1, Tokushima 770 8506, Japan, Tel: +81-88-656-7270; E-mail: matsuo.yoshinori@tokushima-u.ac.jp

Received June 15, 2016; Accepted July 20, 2016; Published July 26, 2016

Citation: Yamamoto Y, Watanabe T, Nakamura M, Kakubayashi N, Saito $Y$ et al. (2016) Epigenetics Evolution and Replacement Histones: Evolutionary Changes at Drosophila H4r. J Phylogenetics Evol Biol 4: 170. doi:10.4172/23299002.1000170

Copyright: (c) 2016 Yamamoto $\mathrm{Y}$, et al. This is an open-access article distributed under the terms of the Creative Commons Attribution License, which permits unrestricted use, distribution, and reproduction in any medium, provided the original author and source are credited. 


\section{5'-region}

Coding region

\section{3'-region}

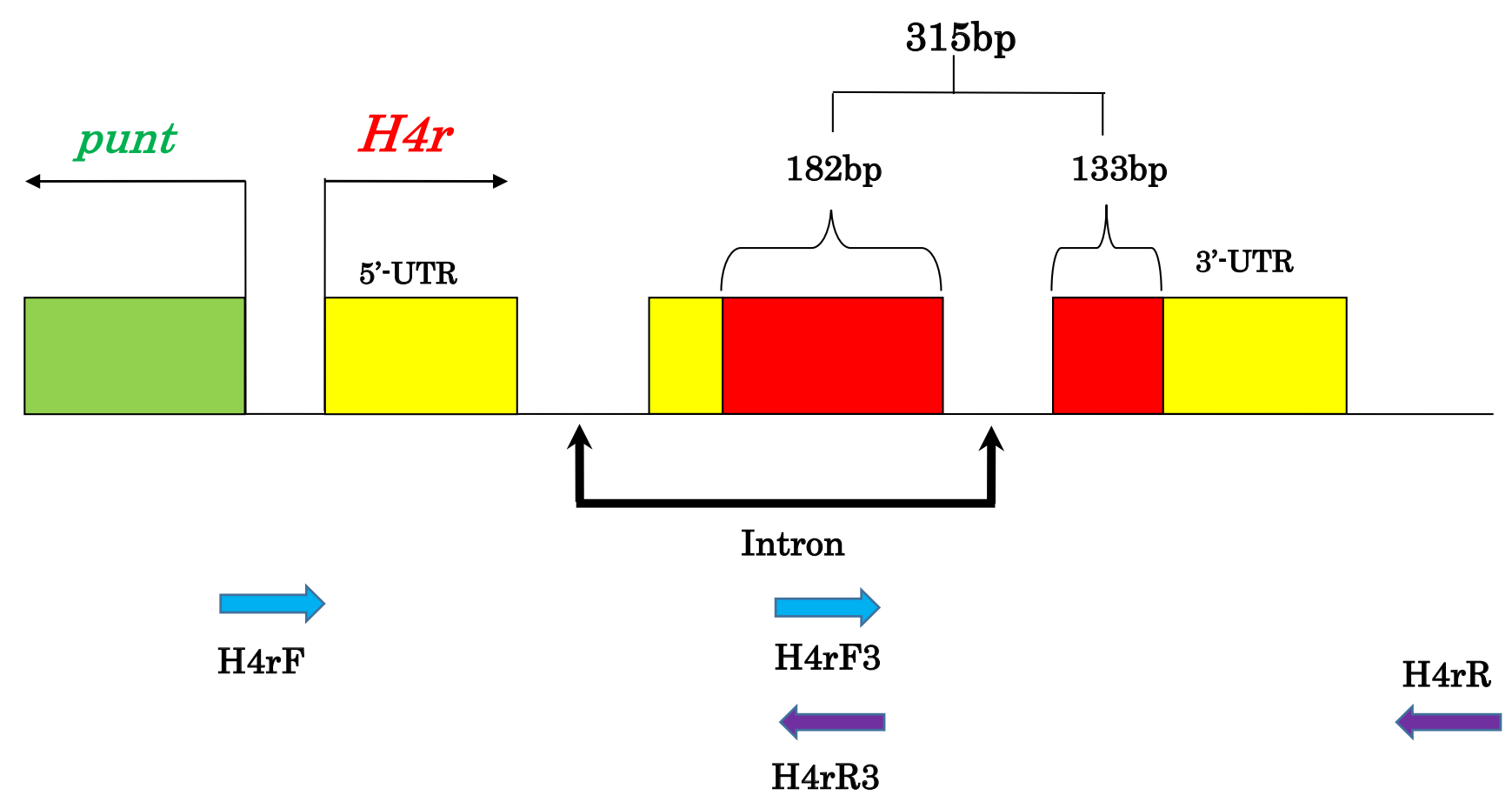

Figure 1: Gene structure of H4r in 14 Drosophila species and sequencing strategy. The locations of PCR primers used for cloning and sequencing are indicated by arrows.

in Figure 1. The primers used for sequencing were 5'-GTTCTCAAGCAGGT-3' (U-19), 5'-CAGGAAACAGCTATGAC-3' (Reverse), 5'-AAGCGTATCTCTGG-3' ( H4rF3), and 5'-CCAGAGATACGCTT-3' ( H4rR3).

\section{Data analysis}

The nucleotide sequence of the replacement histone 4 gene (H4r) from each of three Drosophila species (D. mauritiana, D. erecta, and D. orena) has been deposited into the DNA Data Bank of Japan (DDBJ). The accession numbers for the $\mathrm{H} 4 \mathrm{r}$ genes from $D$. mauritiana, D. erecta, and D. orena are LC127192, LC127193 and LC127194, respectively (Table 1). A second clone from D. orena was also sequenced, but not used for analysis; this clone differed from the other clone at only two nucleotide sites in the 3' region. Table 1 lists all 14 Drosophila species compared here; the DNA sequences of histone genes from the 11 other Drosophila species were obtained from either FlyBase (FB2015-01) or DDBJ (Table 1). Clustal W (ver 2.1) [29] available from DDBJ was used for multiple sequence alignment.

\section{Results}

\section{Genome organization of $\mathbf{H} 4 \mathbf{r}$ genes in fourteen Drosophila species}

Genomic arrangement of each H4r gene from the 14 Drosophila species is shown diagrammatically in Figure 2. For 11 of the 14 species, the $\mathrm{H} 4 \mathrm{r}$ gene was located between a punt gene and a Cep78K gene. The punt gene encodes a transforming growth factor-beta receptor with protein kinase activity. The Cep78K gene encodes a centrosomal protein of $78 \mathrm{KDa}$. In these 11 species, the transcriptional orientation of the H4r gene is head-to-head relative to punt and tail-to-tail relative to Cep78K. In eight closely related species, the length of spacers between these three genes was highly similar. The spacer between punt and the $\mathrm{H} 4 \mathrm{r}$ gene was only slightly longer in D. pseudoobscura and D. persimilis than in the other eight, but it was much longer in D. willistoni. For three more distantly related species (D. mojavensis, D. virilis, and $D$. grimshawi), punt could not be found upstream of $\mathrm{H} 4 \mathrm{r}$ gene. In $D$. virilis and D. grimshawi, another gene containing a MADF (myb/SANTlike domain in Adf-1) domain was found upstream of the H4r gene. These findings suggested that a head-to-head pairing of the $\mathrm{H} 4 \mathrm{r}$ and punt genes was not always necessary for proper expression of $\mathrm{H} 4 \mathrm{r}$; notably, head-to-head pairing is necessary for proper expression of the replication-dependent genes of $\mathrm{H} 3$ and $\mathrm{H} 4$ [30]. A Cep78 K gene was located downstream of $\mathrm{H} 4 \mathrm{r}$ in all 14 species, and the intergenic spacing was similar length in all species. Because of the tail-to-tail arrangement, it was not clear whether a Cep78K gene positioned a short distance downstream of $\mathrm{H} 4 \mathrm{r}$ is necessary for proper $\mathrm{H} 4 \mathrm{r}$ expression.

\section{Comparative analysis of the coding regions for $\mathrm{H} 4$ and $\mathrm{H} 4 \mathrm{r}$} genes in Drosophila

The amino acid sequences deduced from the $\mathrm{H} 4$ and $\mathrm{H} 4 \mathrm{r}$ nucleotide sequences were aligned for all 14 Drosophila species (supplementary data). Of 28 histone 4 genes, 26 showed identical predicted amino acid sequences (consensus sequence) except for two variant $\mathrm{H} 4$ genes, one each in D. sechelia and in D. willistoni (supplementary data). In these two H4 variants, only one (D.willistoni) or two (D. sechelia) amino acid sites differed from the consensus sequence. Notably, the amino acid sequences of $\mathrm{H} 4 \mathrm{r}$ homologs were highly conserved; there were no amino acid substitutions at any site among the 14 Drosophila 
species. This finding suggested that the function of H4r gene was very important and was expected to be the same or similar to that of $\mathrm{H} 4$

Although the amino acid sequences deduced from these genes were identical for all H4r homologs, the nucleotide sequences encoding these amino acids differed among all the genes; no two nucleotide sequences were identical. This means that there were cases in which the nucleotide sequence at synonymous sites differed. Codon usage bias for these genes is shown in Figure 3. For each histone gene, the total codon usage, the number of codon used summed over Drosophila species, was calculated. Although the difference of codon usage for the gene among species is known, a comparison for the gene is possible using the data from the same species. A bias in codon usage was observed for most amino acids, as has been previously reported for other genes [31-32]. However, any difference between two histone types in codon usage was the issue investigated here (Table 2). Although no significant difference in codon usage was observed between the two histone types for six amino acids (His, Asn, Pro, Ser, Tyr and Phe), highly significant differences were found for six other amino acids (Thr, Gly, Arg, Lys, Asp, and Gln). Additionally, moderate differences were found for five other amino acids (Leu, Ala, Val, Glu and Ile). To determine the distribution of codon bias, the codon usage at each amino acid site was listed for two histone 4 genes (Figure 4). At several specific sites, codon usage differed notably between these two histone genes. For example, there was an extreme difference of codon bias at two Lys sites, Lys5 ( $\chi^{2}$ d.f. $=1=24.3, \mathrm{P}<0.001)$ and Lys20 $\left(\chi_{\text {d.f. }=1}^{2}=17.4, \mathrm{P}<0.001\right)$; however, no such difference was observed at other Lys sites. Similar site-specify codon bias was also evident for Arg, Gly and Leu. Whether these specific sites that exhibited biased codon usage are subject to histone modification is a matter of interest. A possible relationship between codon usage bias and histone modification $[7,33]$ is indicated in Figure 4. Several sites that exhibit codon biased (e.g., Lys 5 and Lys 20) seemed to have strong connections to histone modification. Thus the codon usage could be related to the functional difference between $\mathrm{H} 4$ and $\mathrm{H} 4 \mathrm{r}$ genes.

The GC content at the 3rd codon position in the histone 4 genes (H4 and H4r) for the 14 Drosophila species is depicted in Figure 5. Although variability in GC content among the species was observed, the GC content of the H4r gene was higher than that of the $\mathrm{H} 4$ gene in each species studied. This finding indicated that $\mathrm{G}$ or $\mathrm{C}$ was used more frequently at synonymous sites in the $\mathrm{H} 4 \mathrm{r}$ gene than in the $\mathrm{H} 4$ gene.

Conserved sequences in the 5'-region, the intron, and the 3'-region of $\mathrm{H} 4 \mathrm{r}$ genes

Regions upstream of the H4r genes were compared among seven species in the melanogaster group (Figure 6). The spacer between the $\mathrm{H} 4 \mathrm{r}$ and punt genes in each of these species was only 50 or $51 \mathrm{bp}$ long; in contrast, this spacer length between genes was not as strictly conserved relative to the more distantly related species (Figures 2 and 6). Notably, nine base pairs (AGGGCTGGT) upstream of the transcription start site for punt and seven base pair (ATACTAG) in the middle of spacer were relatively conserved among the melanogaster subgroup species, but not the more distantly related species. Thus in the 5'-region of the $\mathrm{H} 4 \mathrm{r}$ gene, there seemed to be no signal sequence that has been conserved strongly as is the case for $\mathrm{H} 3-\mathrm{H} 4$ gene pair [23].

Additionally, the second half of the first intron was relatively conserved among the seven melanogaster subgroup species (Figure 7), although it was not conserved in the distantly related species.

Regions downstream of the H4r genes were also compared among the seven melanogaster subgroup species (Figure 8). The spacer between $\mathrm{H} 4 \mathrm{r}$ gene and CEP78K gene was short (60 $~ 96 \mathrm{bp})$ and relatively constant length even in the distantly related species (Figure 2). However, no conserved sequences were found in this spacer region.
-D. melanogaster

- D. simulans

- D. sechellia

- D. mauritiana

- D. yakuba

- D. erecta

- D. orena

- D. ananassae

- D. pseudoobscura

- D. persimilis

- D. willistoni

- D. mojavensis

- D. virilis

- D. grimshawi
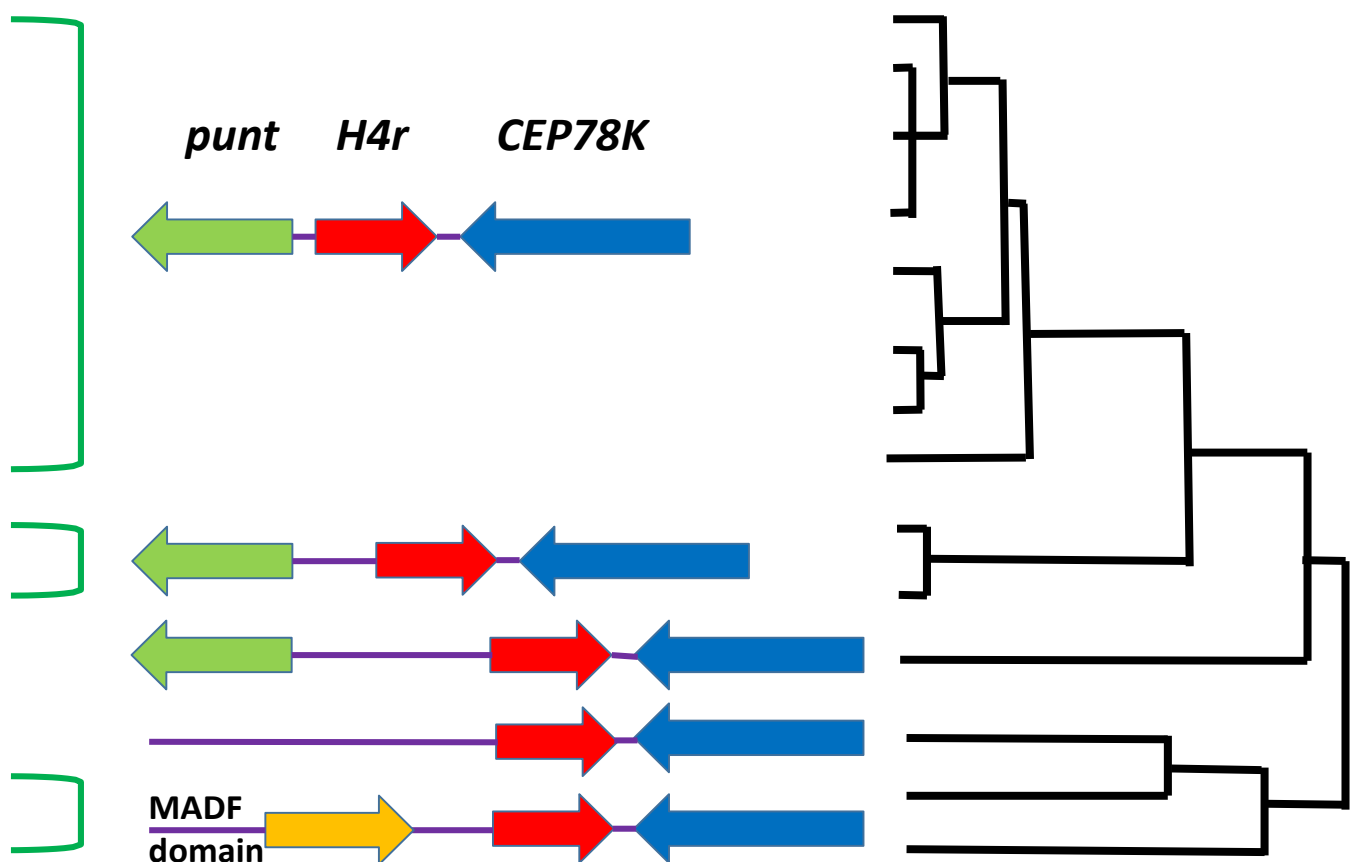

Figure 2: Gene arrangement for punt (green), H4r (red), and CENP78K (blue) in Drosophila genomes. Orientation of transcription is indicated by arrows. Phylogenetic relationship of the 14 Drosophila species is indicated at right side of the figure. 


\begin{tabular}{|c|c|c|}
\hline Species & H4 gene & H4r gene \\
\hline D. melanogaster & X14215 (DDBJ) & CG3379 (FlyBase) \\
\hline D. simulans & $\mathrm{AB} 055959$ (DDBJ) & GD18968 (FlyBase) \\
\hline D. sechellia & $\mathrm{AB} 105183$ (DDBJ) & GM24174 (FlyBase) \\
\hline D. mauritiana & $\mathrm{AB} 105182$ (DDBJ) & LC127192 (DDBJ) \\
\hline D. yakuba & $\mathrm{AB} 073635$ (DDBJ) & GE24247 (FlyBase) \\
\hline D. erecta & $\mathrm{AB} 073634$ (DDBJ) & LC127193 (DDBJ) \\
\hline D. orena & $\mathrm{AB} 105181$ (DDBJ) & LC127194 (DDBJ) \\
\hline D. ananassae & GF19278 (FlyBase) & GF17447 (FlyBase) \\
\hline D. pseudoobscura & AB249651 (DDBJ) & GA17414 (FlyBase) \\
\hline$D$. persimilis & GL17799 (FlyBase) & GL24413 (FlyBase) \\
\hline$D$. willistoni & GK10375 (FlyBase) & GK12810 (FlyBase) \\
\hline D. mojavensis & Gl13912 (FlyBase) & Gl10680 (FlyBase) \\
\hline D. virilis & GJ23527 (FlyBase) & GJ24352 (FlyBase) \\
\hline D. grimshawi & GH23501 (FlyBase) & GH18656 (FlyBase) \\
\hline
\end{tabular}

Table 1: Analysis of the $\mathrm{H} 4$ and $\mathrm{H} 4 \mathrm{r}$ genes in 14 Drosophila species. Nucleotide sequence data were obtained from FlyBase (annotation ID) or DDBJ (accession number).

\begin{tabular}{|c|c|c|}
\hline Amino Acid & $\chi^{2}$ & d.f. \\
\hline Thr & $53.04^{* * *}$ & 3 \\
\hline Gly & $33.24^{* *}$ & 3 \\
\hline Arg & $43.60^{* * *}$ & 5 \\
\hline Lys & $17.96^{* * *}$ & 1 \\
\hline Leu & $17.12^{* *}$ & 5 \\
\hline Ala & $11.61^{* *}$ & 3 \\
\hline His & 1.2 & 1 \\
\hline Val & $12.82^{* *}$ & 3 \\
\hline Asp & $21.26^{* * *}$ & 1 \\
\hline Asn & $4.08^{*}$ & 1 \\
\hline Ile & $12.0^{* *}$ & 2 \\
\hline Gln & $21.32^{* * *}$ & 1 \\
\hline Pro & 3.87 & 3 \\
\hline Ser & 0.67 & $2^{\mathrm{a}}$ \\
\hline Tyr & 0 & 1 \\
\hline Glu & $10.32^{* *}$ & 1 \\
\hline Phe & 1.79 & 1 \\
\hline
\end{tabular}

***: $\mathrm{P}<0.001 ;{ }^{* *}: \mathrm{P}<0.01 ;{ }^{*}: \mathrm{P}<0.05 ;$ a: The expected value which is smaller than one is combined with the next smallest value.

Table 2: $\mathrm{X}^{2}$ tests for codon bias of the $\mathrm{H} 4$ and $\mathrm{H} 4 \mathrm{r}$ genes in 14 Drosophila species.

\section{Discussion}

\section{Gene structure, control signal and function of $\mathrm{H} 4 \mathrm{r}$}

In many cases, the gene pair encoding replication-dependent histones (e.g., H3-H4 or H2A-H2B in Drosophila) has a head-tohead orientation [16,24-25], and transcription of two genes is coregulated [30]. In the closely related species of the melanogaster group, the H4r and punt genes were arranged in a head-to-head orientation and only about $50 \mathrm{bp}$ apart. In the more distantly related species, however, the length of the spacer between two genes was not conserved. In D.mojavensis, D. virilis, and D. grimshawi, punt was not located near the $\mathrm{H} 4 \mathrm{r}$ gene and was located in another region. This finding suggested that the H4r-punt pairing did not have some highly conserved functional significance. However, the possibility cannot be excluded that this pairing of genes has a function in the melanogaster group. CEP78K, which encodes a centriole protein, was located downstream of the $\mathrm{H} 4 \mathrm{r}$ gene in all 14 species studied; however, the tail-to-tail orientation and the lack of a signal sequence element in the spacer suggested that these two genes were not co-regulated.
The gene structures of two types of histone gene were quite different from each other. A multiple-copy gene structure seemed to be favorable for producing a large amount of protein in a short period of time, while a single-copy structure seemed to be favorable for fine-tuning the expression level of the H4r gene. Additionally, an exon-intron structure allows for the production of multiple kinds of transcripts from a single transcription unit. The transcription termination signals are a hair-pin loop structure and a poly (A) signal for $\mathrm{H} 4$ gene and H4r gene, respectively [26,34-35]. A protein that binds to the hairpin loop structure is part of transcription complex; this complex facilitates the coordination between histone mRNA transcription and DNA replication [30].

As the amino acid sequence of $\mathrm{H} 4 \mathrm{r}$ was identical to that of most $\mathrm{H} 4$ copies; this identity is the reason that $\mathrm{H} 4 \mathrm{r}$ is not considering a histone 'variant'. Because of the conserved protein, even a single amino acid substitution has not been tolerated during the evolution of $\mathrm{H} 4$ and $\mathrm{H} 4 \mathrm{r}$. Although the exact function of $\mathrm{H} 4 \mathrm{r}$ is not clear, the strong conservation of this protein among the 14 Drosophila species suggested that this protein has an important role in chromatin remodeling. Therefore, $\mathrm{H} 4 \mathrm{r}$, like $\mathrm{H} 3.3$ and $\mathrm{H} 2 \mathrm{AvD}$, is expected to play a significant role in histone replacement. It seemed to be a key for chromatin remodeling that non modified or differently modified histones replace with the RDHs. Therefore the mechanisms which produce different control of expression will be important rather than the difference of primary structure.

In the region upstream of $\mathrm{H} 4 \mathrm{r}$ gene, no transcriptional control signals were found. In addition, based on FlyBase data, the expression profile for the H4r gene in Drosophila (FB2015-01) indicated that the transcripts were observed at most developmental stages and at similar expression levels. Therefore, transcription may not be a critical step in the control of the H4r gene. Post-transcriptional or post-translational processes (e.g., histone modification) might be important for chromatin remodeling.

\section{Codon usage and histone modification}

The codon usage of Drosophila histone genes has been investigated for many species [19,36-38]. Mechanisms of generating bias for codon usage such as selection-mutation balance, the effect of population size and bias for mutation pattern have been extensively studied $[23,39-44]$. The overall codon usage did not differ greatly between the two histone 4 genes, $\mathrm{H} 4$ and H4r. However, a site-by-site analysis of codon bias showed that some sites did exhibit substantial difference. Sites exhibiting moderate bias were not common. Thus, codon usage at particular sites did differ between $\mathrm{H} 4$ and H4r. The relationship between codon usage and histone modification was not clear, but codon bias is one characteristic difference between the two types of histone. It is generally recognized that histone modifications occur posttranslationally $[1,45]$. However, some types of histone modification, e.g., methylation of H3 Lys 9, occur during translation [46]. This finding suggests the possibility that differences in codon usage could affect the efficiency of some histone modifications. This finding also means that the differences in codon usage between the two types of histone could have functional significance.

The differences of gene structure and codon usage observed between two types of histone genes may be relevant to the respective functions. Therefore, the evolutionary mechanism for developing 
Citation: Yamamoto Y, Watanabe T, Nakamura M, Kakubayashi N, Saito Y, et al. (2016) Epigenetics Evolution and Replacement Histones: Evolutionary Changes at Drosophila H4r. J Phylogenetics Evol Biol 4: 170. doi:10.4172/2329-9002.1000170
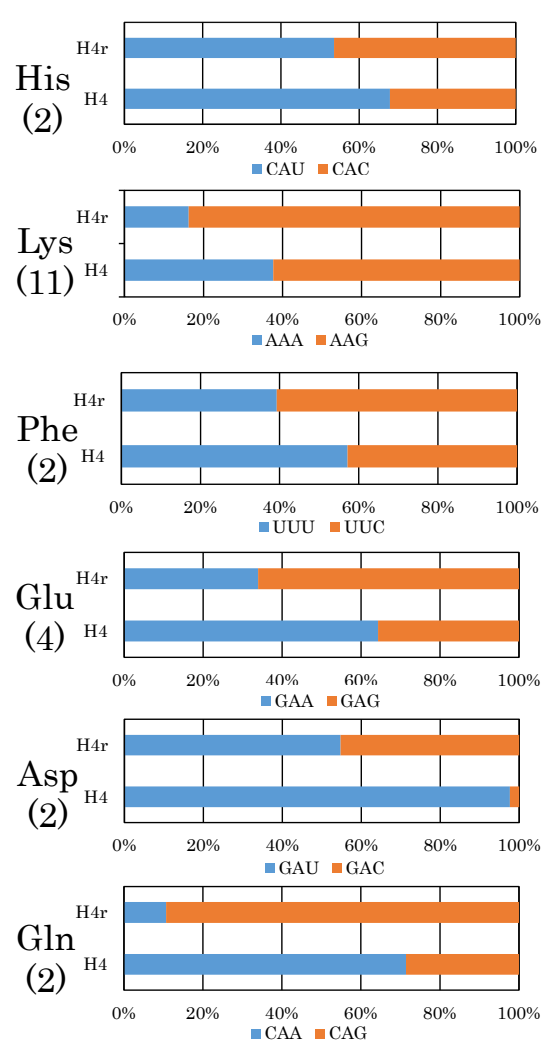
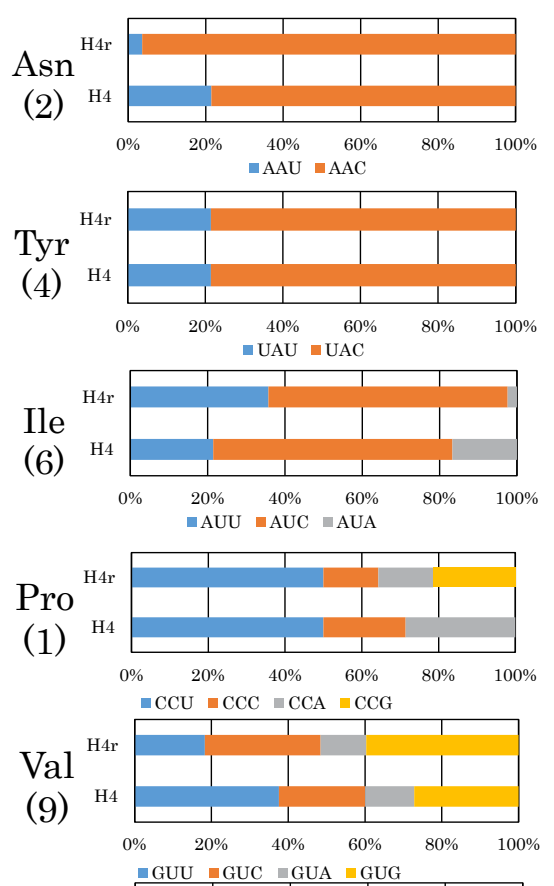

Thr

(8)

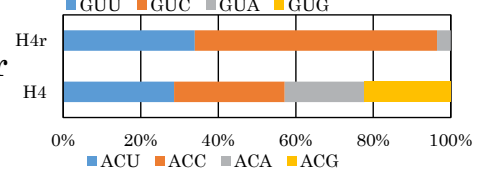

Gly

(17)

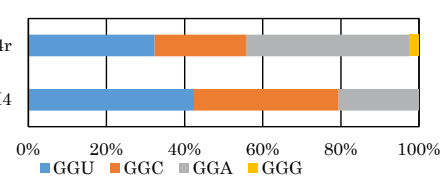

Ala

(7)

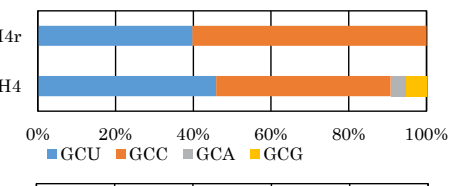

Arg

(14)

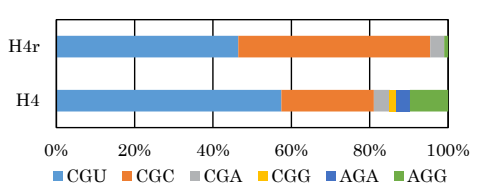

Leu

(8)

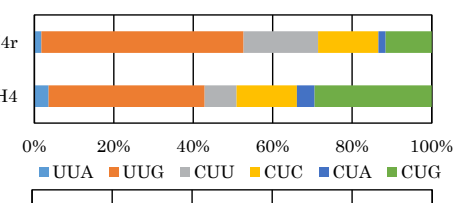

Ser

(1)

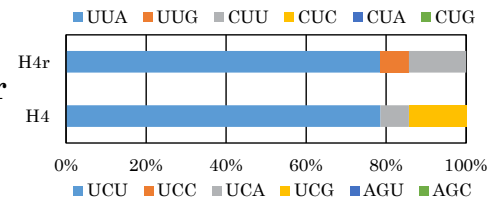

Figure 3: Codon usage of histone genes for $\mathrm{H} 4$ and $\mathrm{H} 4 \mathrm{r}$ in Drosophila. Numbers within the parenthesis indicate the number of constituent amino acids in $\mathrm{H} 4$ and $\mathrm{H} 4 \mathrm{r}$.

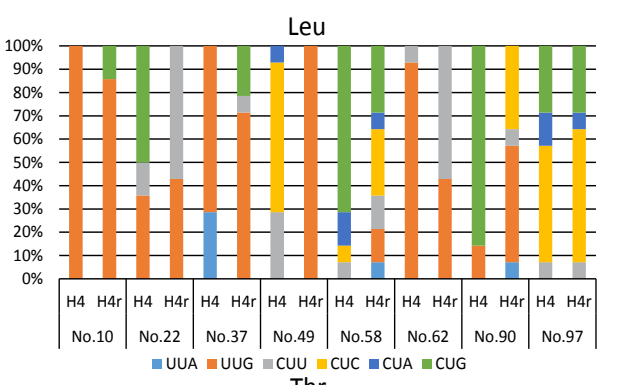

Thr

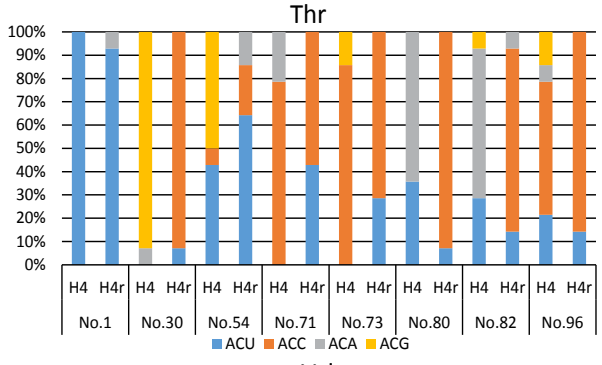

Val

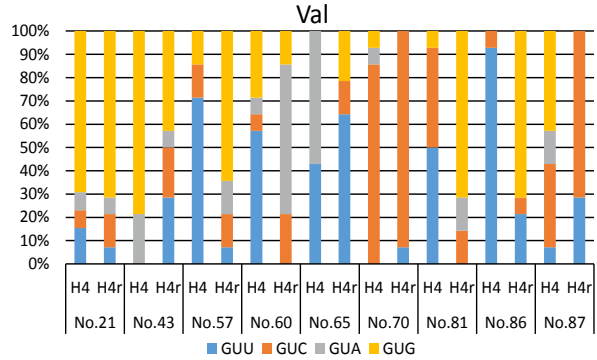

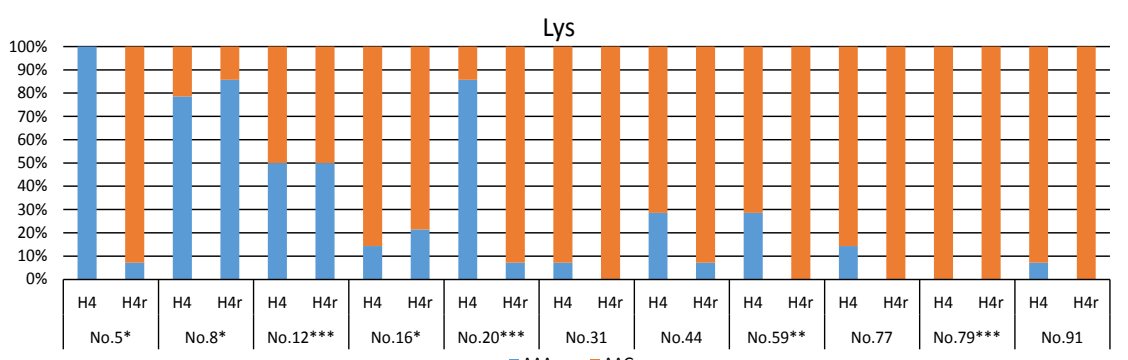

Arg
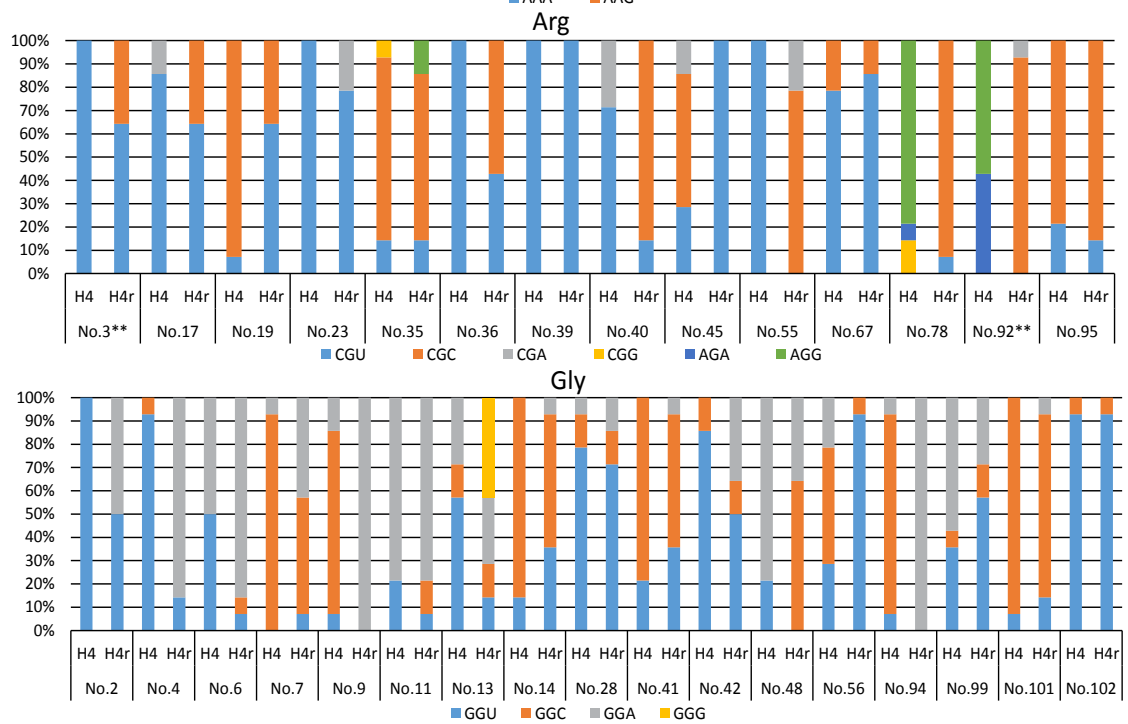
Citation: Yamamoto Y, Watanabe T, Nakamura M, Kakubayashi N, Saito Y, et al. (2016) Epigenetics Evolution and Replacement Histones: Evolutionary Changes at Drosophila H4r. J Phylogenetics Evol Biol 4: 170. doi:10.4172/2329-9002.1000170
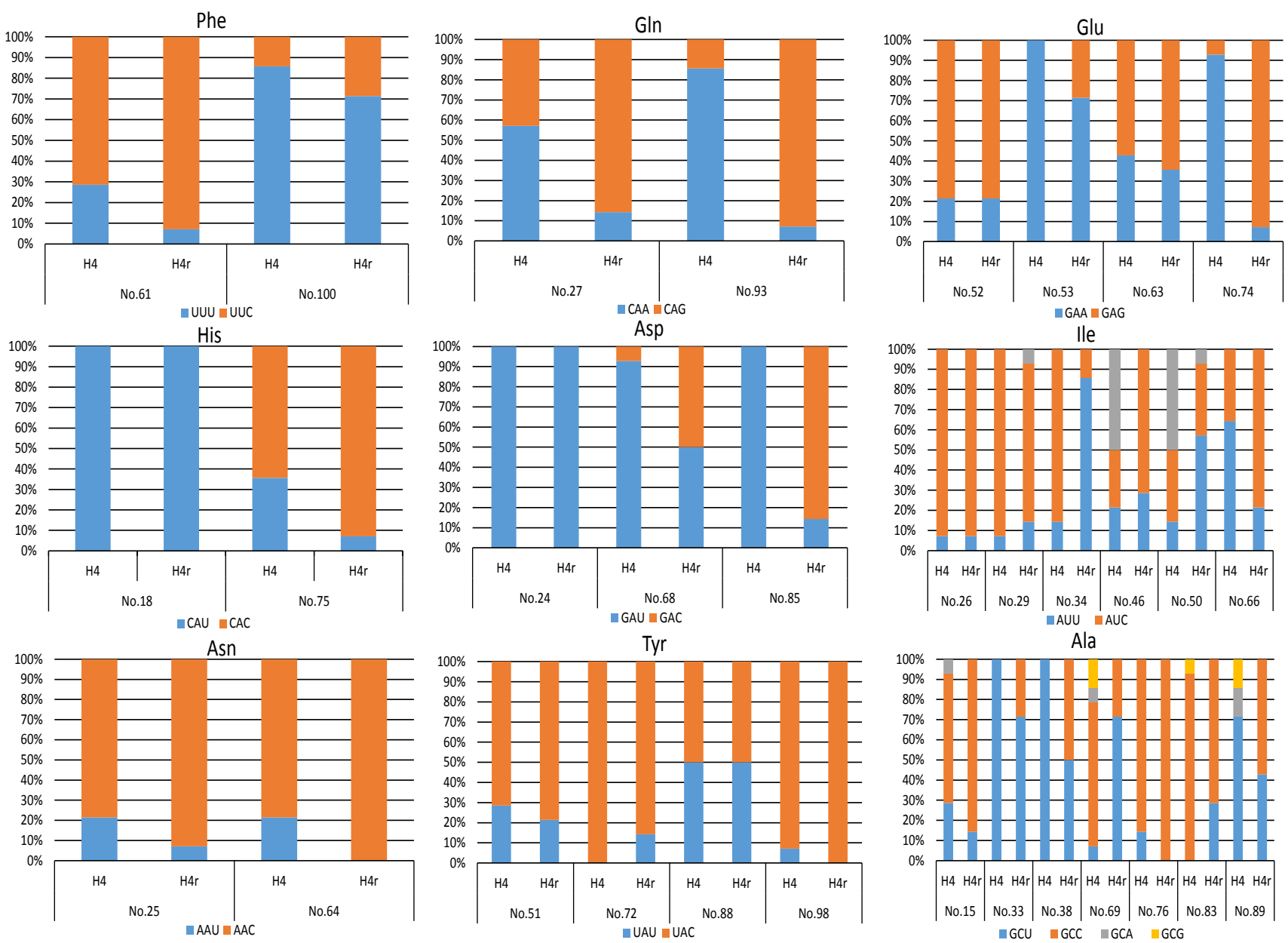

Figure 4: Codon usage at each amino acid site of the respective histone 4 genes in Drosophila. The locations of histone modifications are indicated as follows: *(Acetylation mark), **(Methylation mark) and ${ }^{* * *}$ (Acetylation and methylation mark) after the position number.

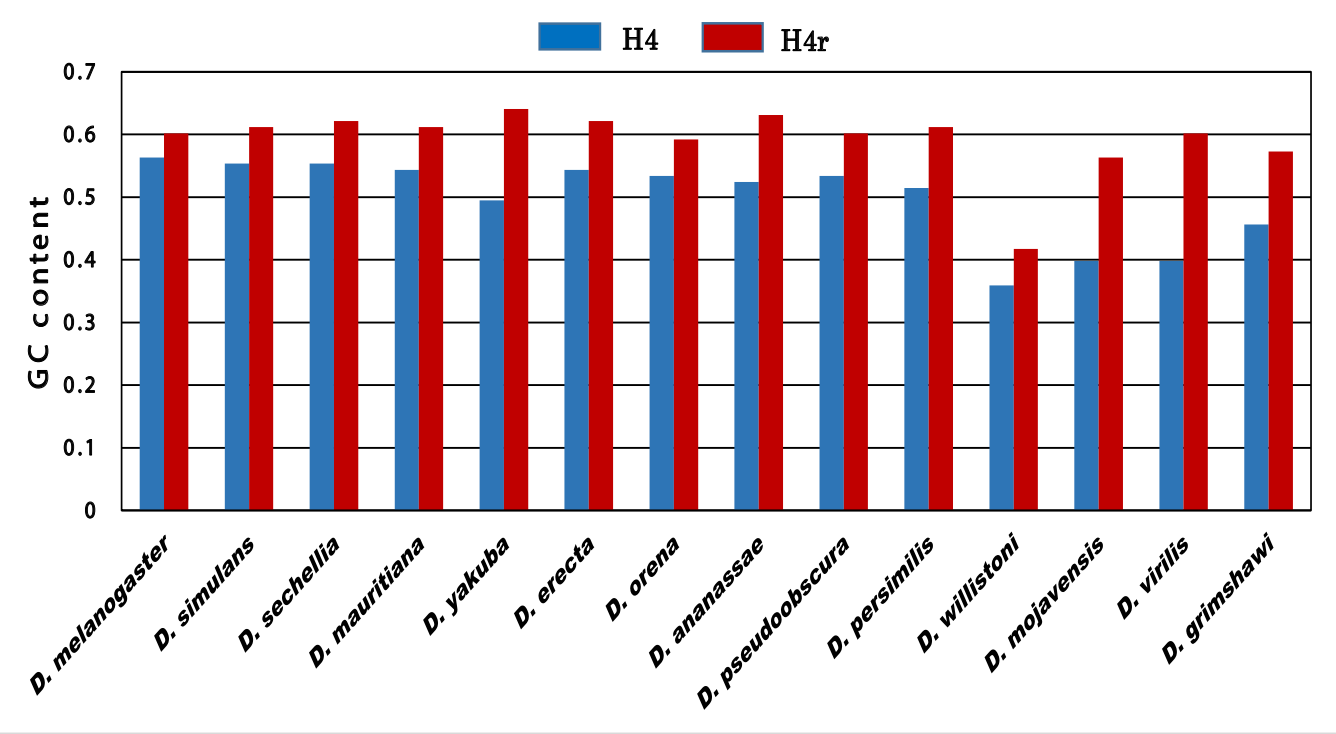

Figure 5: GC content at $3^{\text {rd }}$ codon positions of the $\mathrm{H} 4$ (blue) and $\mathrm{H} 4 \mathrm{r}$ (red) genes in Drosophila. 
Citation: Yamamoto Y, Watanabe T, Nakamura M, Kakubayashi N, Saito Y, et al. (2016) Epigenetics Evolution and Replacement Histones: Evolutionary Changes at Drosophila H4r. J Phylogenetics Evol Biol 4: 170. doi:10.4172/2329-9002.1000170

Page 7 of 9

D. simulans_H4r

D. sechel/ia_H4r

D. mauritiana_H4r

D. melanogaster_H4r

D. erecta_H4r

D. orena_H4r

D. yakuba_H4r

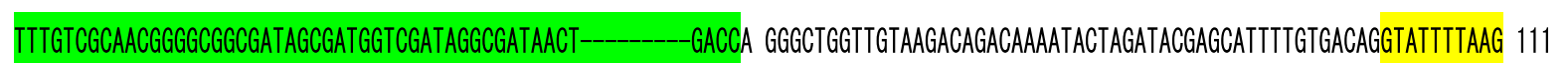

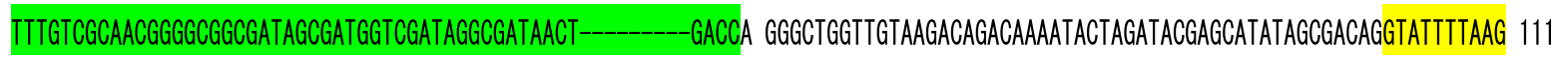
TTTGTCGCAACGGGGCGGCGATAGCGAAGGCCGATAGGCGATAACT--_---GACCA GGGCTGGTTGTAAGACAGACAAAATACTAGATACGAGCATTTTGCGACAGGTATtTTAAG 111 TTTGTCGCAACGGGGCGGCGATAGGGATGGCCGATAGGGGATAACT--_--GACCA GgGCTGGTAGTGAGACAATAGAAATACTAGATACGCGCATTtTGCGAAAGGTATtTTGAG 111 ITTGTCGCAACGGGGCGGCGATAGCGATGGCCGATAGCCGATAACCTCAACCAGGGACCA GGGCTGGTTATAAGACAACTAAAATACTAGATATGAGCATtGGGCGgTAGGTATttTAaG 120 ITTGTCGCAACGGGGCGGGGATAGCGATGGCCGATAGCCGATAACCTCAACT---GaCGA GGGCTGGTTATAAGAGAACTAAAATACTAGGTATGAGCATtGCGCGgAagGTATttTAAG 117 TTTGTAGCGACGgGGCGGCGATAGCGATGGCCGATAGCCGATAACCTCAACT---GACCA GGGCTGGT-GTAAGATAACGAATATACTAGGTATGAGCATTCCGCGGCAGGTATTtTGAG 116 ***** $* * * * * * * * * * * * * * * * * * * * * * * * * * * * * * * * * * * * \quad \quad * * * * * * * * * * * * \quad * * * * * \quad * * * * * * * * * * * * * * * * \quad * * \quad * * * * * * * * * * *$

D. simulans_H4r GTGTGCGCAGTCTGGTCACGCTCACAAACAA-TTTTCTGGAGTtTTCTTGATGTGAGTTT TGTTGCTGAGAAAATTAAGTAAATAATAGGAAA--ATCGGACAACAAA-GTTTGTT 223

D. sechel/ia_H4r GTGTGCGCAGTCTGGTCACGCTCACAAACAA-TTTTCTGGAGTTTTCTTGATGTGAGTTT TGTTGCTGAGAAAATTAAGTAAATAATAGGAAA--ATCGGACAACAAA-----GTTTGTT 223

D. mauritiana_H4r GTGTGCGCAGTCTGGTCACGCTCACAAACAA-TTtTCTGGAGTTTTCTTGATGTGAGTtT TGTTGCTGAGAAAATTAAGTAAATAATAGGAAA--ATTGGACAATAAA-----gTTCGTT 223 D. melanogaster_H4r GTGTGCGAGATCTGGTCACGCTCACAAGCAA-TTtTCTGGAGATTTCTTGAAGTGAGTTT TGTTGCTGAGAAATTTAAGTAAATAATAGAAGA--ATCGGTCAACAAA-----GTTTTTT 223 D. erecta_H4r GTGTGTGAaGTCTGGTCACGCTCACAAACAAATTTTCTGGATTTTTCTTGAGGTGAGTGT TGCTGCTAAGCATTATAAGTAAATAATAGGAAA--AACGAACAACAAAAACCAGTTTGCT 238 D. orena_H4r GTGTGTGAAGTCTGGTCACGCTCACAAACAAATTtTCTGGATtTTTCTTAAGGTGAGTGT TGCTGCTGAGCATTATAAGTAAATAATAGGAAACAAACGAGCAACAAAAACCAGTCTGCT 237 D. yakuba_H4r GTGTCTGAAGTCTGGTCACGCTCACTAACAA-TTTTCTGGATTTTTCTTGAGGTGAGTGT TGCTGCTCGGCAAATTAAGTAAATAATAGAAAA---AT-AACAAAAGAAAACACTTTTTT 231

Figure 6: Nucleotide sequence comparison of the 5' flanking region of $\mathrm{H} 4 \mathrm{r}$ genes in Drosophila. Nucleotide sequences of the melanogaster group are aligned. Asterisk under the sequence indicates identical nucleotides at the respective sites in seven Drosophila species. The regions are shown by the same colors as indicated in Figure 1.

D. simulans_H4r

D. sechel/ia_H4r

D. mauritiana_H4r

D. melanogaster_H4r

D. erecta_H4r

D. orena_H4r

D. yakuba_H4r

D. simulans_H4r

D. sechel/ia_H4r

D. mauritiana_H4r

D. melanogaster_H4r

D. erecta_H4r

D. orena_H4r

D. yakuba_H4r
ITTGCAAAAA-CATTTAGAAAATAAGTAGTAAACATTCGTTTGCATAAAAACGCACTCAT TAACAACAGTTTTCACCTGTCAAATGA------CCTGTTACCTtTCACCCCCACCAAACA 336 TTTGCAAAAA-CGTTTAGAAAATAAGTAGTAACCATTCTTTTGTATAAAAACACACTTAT TAACAACAGTTTTCACCTGTCAAATGA------CCTGTTACCTTTCACCCCCACCAAACA 336 ITTGCAAAAAACATTAAGAAAATAAGTAGTAACCATTCTTTTGTATAAAAACACACTTAT TAACAACAGTTTTCACCTGTCAAATGA-----CCTGTTACCTTTCACCCCCACCAAACA 337 TGTGCATAAA-CATAAAAAAAATAATTGGAGACCATTATtTTGTATGAAAACAGACTTAT TAACAACAGTTTTCACCTGTCAAATGA------ACGTTTACCTtTCACC-----AAACA 330 AATGCAAAAA-TAT-GAAACAATAAACAGAAACCAAACTTTTGCATTGAAACTCACTTGT -AGCAACAGATtTAACCAGTTAAATTA-----CCTGCTAGTtTTCACCCCCACCAAACA 349 TGTGCAAAAA-TAT-GAAGCAATAAATAAAAGCCAAACTTTTGCATTGAAACTCACTTGT -AACAATAGATTTCACCTGTTAAATTAACATTACCTGCTAGTTTTCACCCCCACCAAACA 354 TGTGCAAGAA-CAT-AAAACAGTAAACAAAAGCCATACTTTTGCATTGTAACGCACTTGT TAAGAACAGATTACACCTGTTAAATTA------CCTGTTACTTtTTACCCCCACCAAACA 343

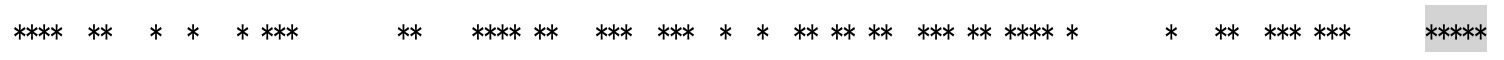

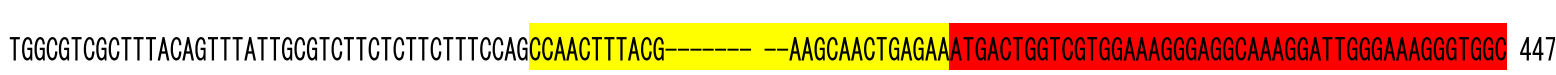

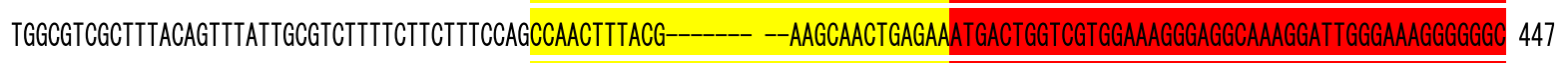

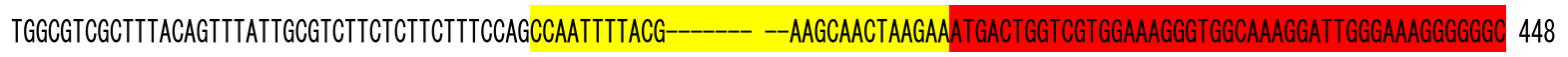

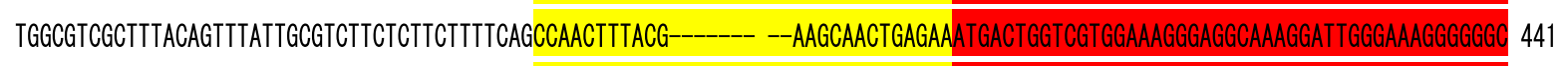
TGGCGTCGCTTCACCGTtTATTGCGTCTTCTCTTCTtTCCAGCGAATtTCACAAAGCAAA GTAAGCAACTAAAAAATGACTGGAGGCGGAAAGGGAGGAAAAGGATTGgGAAAGgGGGGC 469

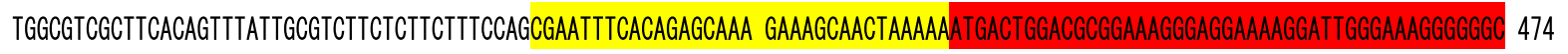

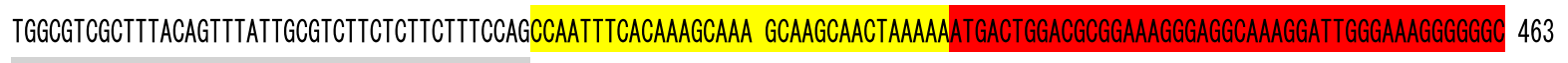
$* * * * * * * * * * * * * * * * * * * * * * * * * * * * * * * * * * * * * * * * * * * * * *$

Figure 7: Nucleotide sequence comparison of the first intron in $\mathrm{H} 4 \mathrm{r}$ genes in seven Drosophila species. Nucleotide sequences of the melanogaster group are aligned. Asterisk under the sequence indicates identical nucleotides at the respective sites in seven Drosophila species. The regions are shown by the same colors as indicated in Figure 1. 
Citation: Yamamoto Y, Watanabe T, Nakamura M, Kakubayashi N, Saito Y, et al. (2016) Epigenetics Evolution and Replacement Histones: Evolutionary Changes at Drosophila H4r. J Phylogenetics Evol Biol 4: 170. doi:10.4172/2329-9002.1000170

\begin{abstract}
D. simulans_H4r
D. sechel/ia_H4r

GGCCAGGGACGCACCCTCTACGGATTTGGGGTTAAGCACGCCCTCTAACCTCCACTTGA TAAATAACCATGGGGTTTAACTTAACTACACTACATTCAAATTACTTGATCCAATTGAAT

D. mauritiana_H4r

CGCCAGGGACGCACCCTCTACGGATTTGGGGGTTAAGCACGCCCTTTAACCTCCACTTGA TAAATAACCA--GAGGTTAACTTAACT-----ACATTCAAATTACTTGATCCAATTGAAT

D. melanogaster_H4r

D. erecta_H4r

CGCCAGGGACGCACCCTTTACGGATTTGGCGGTTAAGCACGCCCTCTAACCTCCACATAA TAAATAACCATGGAG-TTAACTTAACT-----ACATTCAAAATACTTGATCCAATTGAA-

D. orena_H4r

CGCCAGGGACGCACCCTCTACGGTTTTGGCGGTTAAGCACGCCCTCTAACCTCCACTTGA TAAATAACCAAGGGG-TTAACTTAACT--ACATTCAAATTACTTGATCCAATTGAA-

D. yakuba_H4r GCCAGGGACGCACCCTATACGGTTTTGGCGGTTAAGCACGCCCTCTAACCTCCACTTGA TAAATAACCAAGGGG-TTAACTTAACT-----ACATTCAAATTACTTGATCCAATTGAAGGCCAGGGACGCACCCTCTACGGTTTTGGCGGTTAAGCAGGCCGTCTAATCCCCACTTGA TAAATAACCAAGGGG-TTAACTTAACT -ACATTCAAATTACTTGATCCAATTGAA-

$* * * * * * * * * * * * * * * * * * * * * * * * * * * * * * * * * * * * * * * * * * * * * * * * * * * * * * * * * * * * * * * * *$

$* * * * * * * * * * * * *$

$* * * * * * * * * * * * * * * * * * * * * * * * * *$
\end{abstract}

\begin{abstract}
D. simulans_H4r CTTATCTTAATCAGTAGTATTCAACGCAAACTTAACCGAACGCAAATATTTTATAATTCC ACAA-TATACGGACTAAATAAATCAAAAGATATCACATAGAGAGCTCAATATCATACATT
D. seche//ia_H4r TTTATCTTAATCAGTAGTATTCAACGCAAACTTAACCGAACGCAAATATTTTATAATTCC ACAA-TATACGGACTAAATAAATCAAAAGATATCACCTAGAGAGCTCAATATCATACATA

D. mauritiana_H4r CTTATCTTAATCAGTAGTATTCAACGCAAACTTAACCGAACGCAAATATTTTATAATTCC ACAA-TATACGGACTAAATAAATCAAAAGATATCATCTAGAGAGCTCAATATCATACATA

D. melanogaster_H4r ----TCTTAATCAGTAGTATTCAACGCAAACTTAACCGAACGCAAATATTTTATAATTTC ACAA-TATACGGACTAAATAAATCAAAAGATATCACATAGAGAACTCAATATCATGCATA

D. erecta_H4r ----ACTTAATCAGTAGTAT----CGCA--CTTAACCGATCGCAAATATTTTATAATTCC ACAAATATACGGACTAAATAAATCTAAAAATATCACTTAGACAGCTCAAAATC----ATA

D. orena_H4r ---ACTTAATCAGTAGTATTCAACGCAAACTTAACCGATCGCAAATATTTTATAATTCC ACAAATGTACGGACTAAATAAATCTAAAAATATCACGTAGACAGCTCAAAATC----ATA

D. yakuba_H4r ----TCTTAATCAGTAGTATTCAACGCAAACTTAACCGATCGCAAATATTTTATAATTCC ACAAATATACGGACTAAATAAATCAAAAGATATCACGTAGACAGCTCAAAATC----ATA

*************** $\quad * * * * \quad * * * * * * * * * * * * * * * * * * * * * * * * * * * * * * * * * * * * * * * * * * * * * * * * * * * * * * * * * * * * * \quad * * * * * * * * * * * * * \quad * *$
\end{abstract}

\begin{tabular}{|c|c|c|}
\hline D. simulans_H4r & GTGCATCTTGACTTGGG----CGGTT----AATCAT--_--_-- & -_CCAAAAATAAGGTCCAACAAACTCGCTTAGGGAGCACA \\
\hline D. seche//ia_H4r & TGTGCATCTTGACTTGGG----CGGTT----AATCAT--_-_-_-_. & -_-_-_CAAAAATAAGGTCCAACAAACTCGCT \\
\hline D. mauritiana_H4r & TGTGCTTCTTGACTTGGG----CGGTT----AATCAT- & -CCAAAAATAAGGTCCAACAA \\
\hline D. erecta_H4r & CGTGCATCTTGACTTG-----GCGGTT----AATCGTACCTAGCTTATTTGATGATATTT & CACAAAATAATGCTCCAAAAATAAGGTCCAACAAATTCGCTTAGGGA \\
\hline D. orena_H4r & TGTGCATCTTGACTTG-----GCGGTT-----AATCGTACCTAGCTTATTTCATGATATT & CCCAACATAATGCTCAAAAAATAAGATCCAACAAGTTCGCT \\
\hline
\end{tabular}

Figure 8: Nucleotide sequence comparison of the 3 ' flanking region of $H 4 r$ genes in seven Drosophila. Nucleotide sequences of the melanogaster group are aligned. Asterisk under the sequence indicates identical nucleotides at the respective sites in seven Drosophila species. The regions are shown by the same colors as indicated in Figure 1.

diverse gene structures and codon bias might be important for the histone replacement system and chromatin remodeling. To understand the evolution of histone replacement system, other histones variant, such as variants of $\mathrm{H} 3$ and $\mathrm{H} 2 \mathrm{~A}$, should be also investigated.

\section{Conclusions}

In this study, the replacement histone 4 genes from Drosophila are analyzed to investigate the functional differentiation and conservation. The results suggested that the post-transcriptional and posttranslational processes such as histone modifications are important for replacing histones and remodeling chromatin. Evolutionary mechanisms that affect gene structure and codon usage might be important in the emergence of epigenetic systems that depend on replacement histones.

\section{Supplementary Data}

Additional Figure $\mathrm{S} 1$ is available.

\section{Acknowledgement}

This research was supported by a Grant-in-Aid for Scientific Research to Y. M. from the Ministry of Education, Culture, Sports, Science and Technology of Japan.

\section{References}

1. Grunstein M (1997) Histone acetylation in chromatin structure and transcription. Nature 389: 349-352.
2. Kouzarides T (2007) Chromatin modifications and their function. Cell 128: 693-705.

3. Mito Y, Henikoff JG, Henikoff S (2005) Genome-scale profiling of histone H3.3 replacement patterns. Nat Genet 37: 1090-1097.

4. Feng S, Jacobsen SE, Reik W (2010) Epigenetic reprogramming in plant and animal development. Science 330: 622-627.

5. Rothbart SB, Strahl BD (2014) Interpreting the language of histone and DNA modifications. Biochim Biophys Acta 1839: 627-643.

6. Weber CM, Henikoff $S$ (2014) Histone variants: dynamic punctuation in transcription. Genes Dev 28: 672-682.

7. Elliott GO, Murphy KJ, Hayes JJ, Thiriet C (2013) Replication-independent nucleosome exchange is enhanced by local and specific acetylation of histone H4. Nucleic Acids Res 41: 2228-2238.

8. Riddle NC, Minoda A, Kharchenko PV, Alekseyenko AA, Schwartz YB, et al (2011) Plasticity in patterns of histone modifications and chromosomal proteins in Drosophila heterochromatin. Genome Res 21: 147-163.

9. Wang $\mathrm{Cl}$, Alekseyenko AA, LeRoy G, Elia AEH, Gorchakov AA, et al. (2013) ChIP-mass spectrometry captures protein interactions and modified histones associated with dosage compensation in Drosophila. Nat Struct Mol Biol 20: 202-209.

10. Maze I, Noh KM, Soshnev AA, Allis CD (2014) Every amino acid matters: essential contributions of histone variants to mammalian development and disease. Nat Rev Genet 15: 259-271.

11. Isenberg I (1979) Histones. Annu Rev Biochem 48: 159-191.

12. Wells DE (1986) Compilation analysis of histones and histone genes. Nucleic Acids Res 14: r119-r149. 
Citation: Yamamoto Y, Watanabe T, Nakamura M, Kakubayashi N, Saito Y, et al. (2016) Epigenetics Evolution and Replacement Histones: Evolutionary Changes at Drosophila H4r. J Phylogenetics Evol Biol 4: 170. doi:10.4172/2329-9002.1000170

13. Kornberg RD (1977) Structure of chromatin. Annu Rev Biochem 46: 931-954.

14. Fretzin S, Allan BD, Angela van Daal, Elgin SCR (1991) A Drosophila melanogaster $\mathrm{H} 3.3 \mathrm{cDNA}$ encodes a histone variant identical with the vertebrate H3.3. Gene 107: 341-342.

15. Akhmanova A, Miedema K, Hennig W (1996) Identification and characterization of the Drosophila histone H4 replacement gene. FEBS Lett 388: 219-222.

16. Matsuo Y, Yamazaki T (1989) tRNA derived insertion element in histone gene repeating unit of Drosophila melanogaster. Nucleic Acids Res 17: 225-238.

17. Matsuo Y, Yamazaki T (1989) Nucleotide variation and divergence in the histone multigene family in Drosophila melanogaster. Genetics 122: 87-97.

18. Kremer H, Hennig W (1990) Isolation and characterization of a Drosophila hydei histone DNA repeat unit. Nucleic Acids Res 18: 1573-1580.

19. Fitch DHA, Strausbaugh LD (1993) Low codon bias and higher rates of synonymous substitution in Drosophila hydei and D. melanogaster histone genes. Mol Biol Evol 10: 397-413.

20. Tsunemoto K, Matsuo Y (2001) Molecular evolutionary analysis of a histone gene repeating unit from Drosophila simulans. Genes Genet Syst 76: 355-361.

21. Kakita M, Shimizu T, Emoto M, Nagai M, Matsuo Y, et al. (2003) Divergence and heterogeneity of the histone gene repeating units in the Drosophila melanogaster species subgroup. Genes Genet Syst 78: 383-389.

22. Nagoda N, Fukuda A, Nakashima Y, Matsuo Y (2005) Molecular characterization and evolution of the repeating units of histone genes in Drosophila americana: coexistence of quartet and quintet units in a genome. Insect Mol Biol 14:713-717.

23. Matsuo Y (2006) Genomic structure and evolution of the histone gene family in Drosophila. Current Topics in Genetics 2:1-14.

24. Pardue ML, Kedes LH, Weinberg ES, Birnstiel ML (1977) Localization of sequences coding for histone messenger RNA in the chromosomes of Drosophila melanogaster. Chromosoma 63: 135-151.

25. Lifton RP, Goldberg ML, Karp RW, Hogness DS (1977) The organization of the histone genes in Drosophila melanogaster: functional and evolutionary implications. Proc Cold Spring Harbor Symp Quant Biol 42: 1047-1051.

26. Marzluff WF, Wagner EJ, Duronio RJ (2008) Metabolism and regulation of canonical histone mRNAs: life without a poly $(A)$ tail. Nat Rev Genet 9: 843-854.

27. Saiki RK, Scharf S, Faloona F, Mullis KB, Horn GT, et al. (1985) Enzymatic amplification of ß-globin genomic sequences and restriction site analysis for diagnosis of sickle cell anemia. Science 230: 1350-1354.

28. Sanger F, Nicklen S, Coulson AR (1977) DNA sequencing with chain-terminating inhibitors. Proc Natl Acad Sci U S A 74: 5463-5467.

29. Thompson JD, Higgins DG, Gibson TJ (1994) CLUSTAL W: improving the sensitivity of progressive multiple sequence alignment through sequence weighting, position-specific gap penalties and weight matrix choice. Nucleic Acids Res 22: 4673-4680.
30. Salzler HR, Tatomer DC, Malek PY, McDaniel SL, Orlando AN, et al. (2013) A sequence in the Drosophila $\mathrm{H} 3-\mathrm{H} 4$ promoter triggers 1 Histone Locus Body assembly and biosynthesis of replication-coupled histone mRNAs. Dev Cell 24: 623-634.

31. Shields DC, Sharp PM, Higgins DG, Wright W (1988) "Silent" sites in Drosophila genes are not neutral: evidence of selection among synonymous codons. Mol Biol Evol 5: 704-716

32. Sharp PM, Lloyd AT (1993) Codon usage. In 'An atlas of Drosophila genes' by G. Maroni. Oxford University Press, New York.

33. Li KK, Luo C, Wang D, Jiang H, Zheng YG (2012) Chemical and biochemical approaches in the study of histone methylation and demethylation. Med Res Rev 32: 815-867.

34. Schumperli D (1986) Cell-cycle regulation of histone gene expression. Cell 45 471-472.

35. Sabath I, Skrajna A, Yang XC, Dadlez M, Marzluff WF, et al. (2013) 3'-End processing of histone pre-mRNAs in Drosophila: U7 snRNP is associated with FLASH and polyadenylation factors. RNA 19: 1726-1744.

36. Matsuo Y (2000) Evolutionary change of codon usage for the histone gene family in Drosophila melanogaster and Drosophila hydei. Mol Phylogenet Evol 15: 283-291.

37. Matsuo Y (2000) Molecular evolution of the histone 3 multigene family in the Drosophila melanogaster species subgroup. Mol Phylogenet Evol 16: 339-343.

38. Matsuo Y (2003) Evolution of the GC content of the histone 3 gene in seven Drosophila species. Genes Genet Syst 78: 309-318.

39. Bernardi G, Bernardi G (1986) Compositional constraints and genome evolution. J Mol Evol 24: 1-11.

40. Moriyama EN, Hartl DL (1993) Codon usage bias and base composition of nuclear genes in Drosophila. Genetics 134: 847-858.

41. Akashi $H$ (1995) Inferring weak selection from patterns of polymorphism and divergence at "silent" sites in Drosophila DNA. Genetics 139: 1067-1076.

42. Powell JR, Moriyama EN (1997) Evolution of codon usage bias in Drosophila. Proc Natl Acad Sci U S A 94: 7784-7790.

43. Poh YP, Ting CT, Fu HW, Langley CH, Begun DJ (2012) Population genomic analysis of base composition evolution in Drosophila melanogaster. Genome Biol Evol 4: 1245-1255

44. Lawrie DS, Messer PW, Hershberg R, Petrov DA (2013) Strong purifying selection at synonymous sites in D. melanogaster. PLoS Genet 9: e1003527.

45. Jenuwein T, Allis CD (2001) Translating the histone code. Science 293: 1074 1080.

46. Rivera C, Saavedra F, Alvarez F, Diaz-Celis C, Ugalde V, et al. (2015) Methylation of histone $\mathrm{H} 3$ lysine 9 occurs during translation. Nucleic Acids Res 43: 9097-9106. 